\title{
Research Article \\ Convexity of the Set of Fixed Points Generated by Some Control Systems
}

\author{
Vadim Azhmyakov \\ Departamento de Control Automatico, Cinvestav, A.P. 14-740, Avenida Instituto Politecnico Nacional 2508, \\ 07360 Mexico DF, Mexico
}

Correspondence should be addressed to Vadim Azhmyakov, vazhmyakov@ctrl.cinvestav.mx

Received 20 May 2009; Accepted 26 August 2009

Recommended by Andrei Agrachev

We deal with an application of the fixed point theorem for nonexpansive mappings to a class of control systems. We study closed-loop and open-loop controllable dynamical systems governed by ordinary differential equations (ODEs) and establish convexity of the set of trajectories. Solutions to the above ODEs are considered as fixed points of the associated system-operator. If convexity of the set of trajectories is established, this can be used to estimate and approximate the reachable set of dynamical systems under consideration. The estimations/approximations of the above type are important in various engineering applications as, for example, the verification of safety properties.

Copyright (C) 2009 Vadim Azhmyakov. This is an open access article distributed under the Creative Commons Attribution License, which permits unrestricted use, distribution, and reproduction in any medium, provided the original work is properly cited.

\section{Introduction and Motivation}

This paper addresses an application of the well-known fixed point theorem for nonexpansive mappings in Hilbert spaces (see, e.g., [1,2]) to a class of dynamical systems. The main aim of our contribution is to characterize the set of solutions (trajectories) of the dynamical systems under consideration and to establish the convexity property of this set. First, let us consider a nonlinear closed-loop system given by

$$
\begin{gathered}
\dot{x}(t)=f(x(t), u(x(t))), \text { a.e. on }\left[0, t_{f}\right], \\
x(0)=x_{0},
\end{gathered}
$$

where $f: \mathbb{R}^{n} \times \mathbb{R}^{m} \rightarrow \mathbb{R}^{n}$ is Lipschitz continuous in both components. Let $U$ be a compact and convex subset of $\mathbb{R}^{m}$ and consider measurable feedback control functions $u: \mathbb{R}^{n} \rightarrow U$.

Assume that for every such feedback control function $u(\cdot)$, there exists a solution $x^{u}(\cdot)$ of (1.1), for uniqueness conditions and for some constructive existence conditions for systems 
(1.1) we refer to [3, 4]. Given an initial value $x_{0} \in \mathbb{R}^{n}$ and such a feedback control function $u(\cdot)$, the solution of $(1.1)$ is an absolutely continuous function. Let $\mathbb{W}_{n}^{1,1}\left(0, t_{f}\right)$ be the Sobolev space of all absolutely continuous $\mathbb{R}^{n}$-valued functions $y(\cdot)$ such that the derivative $\dot{y}(\cdot)$ exists almost everywhere and belongs to the Lebesgue space $\mathbb{L}_{n}^{1}\left(0, t_{f}\right)$ of all measurable functions $y:\left[0, t_{f}\right] \rightarrow \mathbb{R}^{n}$ with

$$
\|y(\cdot)\|_{\mathbb{L}_{n}^{1}\left(0, t_{f}\right)}=\int_{0}^{t_{f}}\|y(t)\| d t<\infty .
$$

Recall that $\mathbb{W}_{n}^{1,1}\left(0, t_{f}\right)$ equipped with the norm $\|\cdot\|_{\mathbb{W}_{n}^{1,1}\left(0, t_{f}\right)}$, defined by

$$
\|w(\cdot)\|_{\mathbb{W}_{n}^{1,1}\left(0, t_{f}\right)}:=\|w(\cdot)\|_{\mathbb{L}_{n}^{1}\left(0, t_{f}\right)}+\|\dot{w}(\cdot)\|_{\mathbb{L}_{n}^{1}\left(0, t_{f}\right)}
$$

for $w(\cdot) \in \mathbb{W}_{n}^{1,1}\left(0, t_{f}\right)$ is a Banach space. Moreover, $\mathbb{W}_{n}^{1,1}\left(0, t_{f}\right)$ is the completion of the space of all continuously differentiable $\mathbb{R}^{n}$-valued functions $\mathbb{C}_{n}^{1}\left(0, t_{f}\right)$ with respect to the norm $\|\cdot\|_{\mathbb{L}_{n}^{1}\left(0, t_{f}\right)}($ see, e.g., $[5,6])$. The initial value problem (1.1) can also be considered as a problem in the space $\mathbb{W}_{n}^{1,1}\left(0, t_{f}\right)$.

The reachable set $\widetilde{\mathcal{K}}\left(t, x_{0}\right)$ at time $t$ is the set of states of (1.1) which can be reached at time $t$, when starting at $x_{0}$ at time $t=0$, using all possible controls (see, e.g., [7]). That is, $\widetilde{\mathcal{K}}\left(t, x_{0}\right):=\left\{x^{u}(t) \mid u(\cdot) \in \mathbb{L}_{m}^{1}\left(\mathbb{R}^{n}\right), u(x) \in U\right\}$, where $\mathbb{L}_{m}^{1}\left(\mathbb{R}^{n}\right)$ denotes the Lebesgue space of all measurable functions $u: \mathbb{R}^{n} \rightarrow \mathbb{R}^{m}$. We now formulate our standing hypothesis.

(H1) The reachable sets $\widetilde{\mathcal{K}}\left(t, x_{0}\right)$ are contained in an open bounded superset $\Omega \subset \mathbb{R}^{n}$ for $t \in\left[0, t_{f}\right]$.

This is for example the case if $\Omega$ is a positively invariant set for the system (1.1). Recall that a set in the state space is said to be positively invariant for a given dynamical system if any trajectory initiated in this set remains inside the set at all future time instants. Besides, for a dynamical system (1.1) with bounded right-hand side, the reachable set $\widetilde{\mathcal{K}}\left(t, x_{0}\right)$ is trivially bounded.

Given $l \in \mathbb{R}_{+}$, we introduce the space $u_{l}^{f}$ of admissible feedback control functions $u: \Omega \rightarrow U$ as the space of all Lipschitz functions with Lipschitz constants $l_{u} \leq l$ on $\Omega$. Under the above-mentioned boundedness assumption for the reachable set, we can now consider the reachable set $\mathcal{K}\left(t, x_{0}\right)$ of (1.1) with respect to $\mathcal{U}_{l}^{f}$ given by $\mathcal{K}\left(t, x_{0}\right):=\left\{x^{u}(t) \mid u(\cdot) \in \mathcal{U}_{l}^{f}\right\}$. For the given control system (1.1), we address the task of formulating sufficient conditions for the convexity of the reachable set $\mathcal{k}\left(t, x_{0}\right)$ for every $t \in\left[0, t_{f}\right]$. Note that the convexity of the reachable set or the existence of convex approximations for the 1 reachable set bear a close relation to a computational method for determining positively invariant sets, namely, the ellipsoidal technique (see $[8,9]$ ). In this paper, we also derive conditions for the set of trajectories of $(1.1)$ on $\left[0, t_{f}\right]$, that is,

$$
\tau\left(x_{0}\right):=\left\{x^{u}(\cdot):\left[0, t_{f}\right] \longrightarrow \Omega \mid u(\cdot) \in \mathcal{U}_{l}^{f}\right\}
$$

to be convex. The main convexity result for system (1.1) is based on an abstract fixed point theorem for nonexpansive mappings in Hilbert spaces (see, e.g., [1, 2]). For some 
abstract convexity results for nonlinear mappings we refer to [10], for some applications to optimization and optimal control to [10,11]. For an analysis of reachable sets of dynamical systems in an abstract or hybrid setting, see also [8].

While the main topic of our paper is the estimation of reachable sets for closed-loop systems of type (1.1), we also consider open-loop control systems:

$$
\begin{gathered}
\dot{x}(t)=g(x(t), u(t)), \text { a.e. on }\left[0, t_{f}\right], \\
x(0)=x_{0},
\end{gathered}
$$

where $g$ is a Lipschitz continuous function (in both components) and where $u(t)$ belongs to $U$ for $t \in\left[0, t_{f}\right]$. Let $\mathcal{U}:=\left\{u(\cdot) \in \mathbb{L}_{m}^{2}\left(0, t_{f}\right) \mid u(t) \in U\right\}$ be the space of admissible control signals for system (1.5). Here, $\mathbb{L}_{m}^{2}\left(0, t_{f}\right)$ denotes the Lebesgue space of all square-integrable functions $u:\left[0, t_{f}\right] \rightarrow \mathbb{R}^{m}$ with the corresponding norm. It is assumed that for every admissible timedependent control $u(\cdot) \in \mathcal{U}$ system (1.5) has a unique solution $x^{u}(\cdot) \in \mathbb{W}_{n}^{1,1}\left(0, t_{f}\right)$. As for the closed-loop system (1.1), we will obtain estimates for the reachable sets of (1.5) provided the right-hand sides are bounded.

The paper is organized as follows. In Section 2, we provide the necessary definitions and mathematical results. Section 3 contains the convexity result for the sets of trajectories and for reachable sets of the closed-loop control system (1.1). Section 4 discusses overapproximation of reachable sets for some classes of closed-loop and open-loop control systems with bounded right-hand sides. We also use some techniques from optimal control theory to obtain general approximations of convex reachable sets under consideration. In Section 5, we discuss a possible application of our convexity criterion to optimal control problems with constraints. Section 6 summarizes the paper.

\section{Preliminary Results}

We first provide some relevant definitions and facts. Let $x$ and $y$ be two Banach spaces with $x \subset \mathcal{y}$. We say that the space $x$ is compactly embedded in $y$ and write $x_{\hookrightarrow} \mathcal{Y}$, if $\|v\|_{y} \leq$ $c\|v\|_{\mathcal{X}}$ for all $v \in \mathcal{X}$ and each bounded sequence in $\mathcal{X}$ has a convergent subsequence in $\mathcal{Y}$. We recall a special case of the Sobolev Embedding Theorem (cf. [5, 6]) in Proposition 2.1 and list some interpolation properties of Lebesgue spaces (cf. [6]) in Proposition 2.2.

Proposition 2.1. It holds that $\mathbb{W}_{n}^{1,1}\left(0, t_{f}\right) \hookrightarrow_{c} \mathbb{L}_{n}^{2}\left(0, t_{f}\right)$.

Proposition 2.2. If $1 \leq p \leq q \leq \infty$, then $\mathbb{L}_{n}^{q}(\Omega) \subset \mathbb{L}_{n}^{p}(\Omega)$ and

$$
\|v\|_{\mathbb{L}_{n}^{p}(\Omega)} \leq \operatorname{meas}(\Omega)^{1 / p-1 / q}\|v\|_{\mathbb{L}_{n}^{q}(\Omega)}, \quad \forall v \in \mathbb{L}_{n}^{q}(\Omega),
$$

where $1 / \infty$ is understood to be 0 . In particular one has $\mathbb{L}_{n}^{2}\left(0, t_{f}\right) \subset \mathbb{L}_{n}^{1}\left(0, t_{f}\right)$ and, for all functions $y(\cdot) \in \mathbb{L}_{n}^{2}\left(0, t_{f}\right)$,

$$
\|y(\cdot)\|_{\mathbb{L}_{n}^{1}\left(0, t_{f}\right)} \leq \sqrt{t_{f}}\|y(\cdot)\|_{\mathbb{L}_{n}^{2}\left(0, t_{f}\right)}
$$


We now consider the concept of a nonexpansive mapping in Hilbert spaces and present a fundamental fixed point theorem for such mappings in Proposition 2.3 (cf. [1, 2, 12]). Let $C$ be a subset of a Hilbert space $\mathscr{H}$ with norm $\|\cdot\|_{\mathscr{H}}$. A mapping $T: C \rightarrow \mathscr{H}$ is said to be nonexpansive if

$$
\left\|T\left(h_{1}\right)-T\left(h_{2}\right)\right\|_{\mathscr{d}} \leq\left\|h_{1}-h_{2}\right\|_{\mathscr{d}}
$$

holds true for all $h_{1}, h_{2} \in C$.

Proposition 2.3. Let $C$ be a nonempty, closed, and convex subset of a Hilbert space $\mathcal{H}$ and let $T$ be a nonexpansive mapping of $C$ into itself. Then the set $F(T)$ of fixed points of $T$ is nonempty, closed, and convex.

Now, we return to the given control system (1.1) for which we introduce the system operator

$$
P: u_{l}^{f} \times \mathbb{W}_{n}^{1,1}\left(0, t_{f}\right) \longrightarrow \mathcal{U}_{l}^{f} \times \mathbb{W}_{n}^{1,1}\left(0, t_{f}\right)
$$

defined by the following formula:

$$
P(u(\cdot), x(\cdot))(t):=\left(\begin{array}{c}
u(x(t)) \\
x_{0}+\int_{0}^{t} f(x(\tau), u(x(\tau))) d \tau
\end{array}\right) .
$$

Using the Sobolev Embedding Theorem (as stated in Proposition 2.1), we extend $P$ to the operator:

$$
\widetilde{P}: \mathcal{U}_{l}^{f} \times \mathbb{L}_{n}^{2}\left(0, t_{f}\right) \longrightarrow \mathcal{U}_{l}^{f} \times \mathbb{L}_{n}^{2}\left(0, t_{f}\right)
$$

with $\widetilde{P}$ still being given by the right-hand side of formula (2.5).

We now consider the set of admissible feedback controls $\mathfrak{U}_{l}^{f}$, which is contained in $\mathbb{C}_{m}(\Omega)$, as a subset of the space $\mathbb{L}_{m}^{2}(\Omega)$. The following result specifies properties of the set $\mathcal{U}_{l}^{f}$.

Lemma 2.4. The set $\mathcal{U}_{l}^{f}$ is a closed convex subset of the Hilbert space $\mathbb{L}_{m}^{2}(\Omega)$.

Proof. The set $A_{U}$ of all continuous functions $u(\cdot)$ with range in $U$ and the set $A_{l}$ of all Lipschitz continuous functions with Lipschitz constants $l_{u} \leq l$ are both convex subsets of $\mathbb{C}_{m}(\Omega)$. Therefore, the intersection $\mathcal{U}_{l}^{f}:=\mathcal{A}_{U} \cap \mathcal{A}_{l}$ is also convex.

Because of $\mathbb{C}_{m}(\Omega) \subset \mathbb{L}_{m}^{2}(\Omega)$, the set $\mathscr{A}_{l}$ is a closed set in the sense of the supnorm. Hence $A_{l}$ is also closed in the sense of the norm of the space $\mathbb{L}_{m}^{\infty}(\Omega)$. Using Proposition 2.2, we deduce that this set is a closed subset of the space $\mathbb{L}_{m}^{2}(\Omega)$. Now let us consider a sequence $\left\{u_{k}(\cdot)\right\}$ of functions from $\mathcal{u}_{l}^{f}$ such that

$$
\lim _{k \rightarrow \infty}\left\|u_{k}(\cdot)-\widehat{u}(\cdot)\right\|_{\mathbb{L}_{m}^{2}(\Omega)}=0,
$$


where $\widehat{u}(\cdot) \in \mathbb{L}_{m}^{2}(\Omega)$. Then there exists a subsequence $\left\{u_{s}(\cdot)\right\}$ of $\left\{u_{k}(\cdot)\right\}$ satisfying $\lim _{s \rightarrow \infty}\left\|u_{s}(x)-\widehat{u}(x)\right\|_{\mathbb{R}^{m}}=0$ for almost all $x \in \Omega$ (see [13]). The assumption $\widehat{u}(\cdot) \notin \mathcal{U}_{l}^{f}$ implies the existence of a set $\supset \subseteq \Omega$ of positive measure with $\widehat{u}(x) \notin U$ for all $x \in \mathcal{O}$. On the other hand, we have for a fixed $x \in \mathcal{O}$ :

$$
\lim _{s \rightarrow \infty}\left\|u_{s}(x)-\widehat{u}(x)\right\|_{\mathbb{R}^{m}}=0
$$

Since $U$ is a compact set, $\widehat{u}(x)$ belongs to $U$ for the considered $x \in \mathcal{O}$, contradicting our assumption. Thus, we obtain $\widehat{u}(\cdot) \in \mathcal{U}_{l}^{f}$ showing that the set $\mathcal{U}_{l}^{f}$ is closed. The proof is finished.

By Lemma 2.4, the set $\mathcal{U}_{l}^{f} \times \mathbb{L}_{n}^{2}\left(0, t_{f}\right)$ is a closed convex subset of the Hilbert space $\mathscr{H}:=$ $\mathbb{L}_{m}^{2}(\Omega) \times \mathbb{L}_{n}^{2}\left(0, t_{f}\right)$. Note that the scalar product and the norm in this space can be introduced as follows:

$$
\begin{gathered}
\left\langle\left(u_{1}(\cdot), x_{1}(\cdot)\right),\left(u_{2}(\cdot), x_{2}(\cdot)\right)\right\rangle_{\mathscr{d}}:=\left\langle u_{1}(\cdot), u_{2}(\cdot)\right\rangle_{\mathbb{L}_{m}^{2}(\Omega)}+\left\langle x_{1}(\cdot), x_{2}(\cdot)\right\rangle_{\mathbb{L}_{n}^{2}\left(0, t_{f}\right)}, \\
\|(u(\cdot), x(\cdot))\|_{\mathscr{d}}:=\sqrt{\|u(\cdot)\|_{\mathbb{L}_{m}^{2}(\Omega)}^{2}+\|x(\cdot)\|_{\mathbb{L}_{n}^{2}\left(0, t_{f}\right)}^{2}} .
\end{gathered}
$$

Using the triangle inequality and the Schwarz inequality for the Hilbert spaces $\mathbb{L}_{m}^{2}(\Omega)$ and $\mathbb{L}_{n}^{2}\left(0, t_{f}\right)$, one can verify the standard properties of the introduced scalar product and norm.

\section{Convexity Criteria for Reachable Sets of Closed-Loop Systems}

We next state and prove our main result concerning the operator $\widetilde{P}$ from (2.6) and (2.5) under our standing hypothesis (H1). It will be the basis for formulating sufficient conditions for the convexity of the set $\tau\left(x_{0}\right)$ of trajectories and of the reachable set $\mathcal{K}\left(t, x_{0}\right)$.

Theorem 3.1. Assume that $f$ satisfies the Lipschitz condition:

$$
\left\|f\left(x_{1}, u_{1}\right)-f\left(x_{2}, u_{2}\right)\right\| \leq l_{1}\left\|x_{1}-x_{2}\right\|+l_{2}\left\|u_{1}-u_{2}\right\| \quad \forall x_{1}, x_{2} \in \Omega, \forall u_{1}, u_{2} \in U,
$$

where $t_{f} \sqrt{l^{2}+\left(l_{1}+l_{2} l\right)^{2}} \leq 1$. Then the operator $\tilde{P}$ of the corresponding system is nonexpansive, and the set $F(\widetilde{P})$ of fixed points of $\widetilde{P}$ is nonempty closed and convex.

Proof. We claim that $\widetilde{P}$ is a nonexpansive mapping. To see this, consider

$$
\left\|\tilde{P}\left(u_{1}(\cdot), y_{1}(\cdot)\right)-\tilde{P}\left(u_{2}(\cdot), y_{2}(\cdot)\right)\right\|_{\mathscr{d}}^{2}=\left\|u_{1}\left(y_{1}(\cdot)\right)-u_{2}\left(y_{2}(\cdot)\right)\right\|_{\mathbb{L}_{m}^{2}(\Omega)}^{2}+\left\|\int_{0} \delta(\tau) d \tau\right\|_{\mathbb{L}_{n}^{2}\left(0, t_{f}\right)}^{2}
$$


with $\delta(\tau):=f\left(y_{1}(\tau), u_{1}\left(y_{1}(\tau)\right)\right)-f\left(y_{2}(\tau), u_{2}\left(y_{2}(\tau)\right)\right)$ where control functions $u_{1}(\cdot), u_{2}(\cdot)$ are from $\mathbb{L}_{m}^{2}(\Omega)$, and $y_{1}(\cdot), y_{2}(\cdot)$ are elements of $\mathbb{L}_{n}^{2}\left(0, t_{f}\right)$. Consider the second term of the righthand side of (3.2). We obtain

$$
\begin{aligned}
\left\|\int_{0} \delta(\tau) d \tau\right\|_{\mathbb{L}_{n}^{2}\left(0, t_{f}\right)}^{2} & =\int_{0}^{t_{f}}\left\|\int_{0}^{t} \delta(\tau) d \tau\right\|_{\mathbb{R}^{n}}^{2} d t \\
& \leq \int_{0}^{t_{f}}\left(\int_{0}^{t}\|\delta(\tau)\|_{\mathbb{R}^{n}} d \tau\right)^{2} d t \leq \int_{0}^{t_{f}}\left(\int_{0}^{t_{f}}\|\delta(\tau)\|_{\mathbb{R}^{n}} d \tau\right)^{2} d t \\
& =t_{f}\left(\int_{0}^{t_{f}}\|\delta(\tau)\|_{\mathbb{R}^{n}} d \tau\right)^{2} .
\end{aligned}
$$

From (3.3) and from the Lipschitz condition for the function $f$ it follows that

$$
\begin{aligned}
\left\|\int_{0} \delta(\tau) d \tau\right\|_{\mathbb{L}_{n}^{2}\left(0, t_{f}\right)}^{2} & \leq t_{f}\left(l_{1}+l_{2} l\right)^{2}\left(\int_{0}^{t_{f}}\left\|y_{1}(\tau)-y_{2}(\tau)\right\|_{\mathbb{R}^{n}} d \tau\right)^{2} \\
& =t_{f}\left(l_{1}+l_{2} l\right)^{2}\left\|y_{1}(\cdot)-y_{2}(\cdot)\right\|_{\mathbb{L}_{n}^{1}\left(0, t_{f}\right)}^{2} .
\end{aligned}
$$

By Proposition 2.2, we have the following estimation:

$$
\left\|y_{1}(\cdot)-y_{2}(\cdot)\right\|_{\mathbb{L}_{n}^{1}\left(0, t_{f}\right)}^{2} \leq t_{f}\left\|y_{1}(\cdot)-y_{2}(\cdot)\right\|_{\mathbb{L}_{n}^{2}\left(0, t_{f}\right)}^{2}
$$

This fact and inequality (3.6) both imply

$$
\left\|\int_{0} \delta(\tau) d \tau\right\|_{\mathbb{L}_{n}^{2}\left(0, t_{f}\right)}^{2} \leq t_{f}^{2}\left(l_{1}+l_{2} l\right)^{2}\left\|y_{1}(\cdot)-y_{2}(\cdot)\right\|_{\mathbb{L}_{n}^{2}\left(0, t_{f}\right)}^{2} .
$$

Since $t_{f} \sqrt{l^{2}+\left(l_{1}+l_{2} l\right)^{2}} \leq 1$, we finally deduce from Proposition 2.2, and formulas (3.2)-(3.6):

$$
\begin{aligned}
\left\|\tilde{P}\left(u_{1}(\cdot), y_{1}(\cdot)\right)-\tilde{P}\left(u_{2}(\cdot), y_{2}(\cdot)\right)\right\|_{\mathscr{\ell}} & \leq\left[\left\|u_{1}(\cdot)-u_{2}(\cdot)\right\|_{\mathbb{L}_{m}^{2}(\Omega)}^{2}+\left\|y_{1}(\cdot)-y_{2}(\cdot)\right\|_{\mathbb{L}_{n}^{2}\left(0, t_{f}\right)}^{2}\right]^{1 / 2} \\
& =\left\|\left(u_{1}(\cdot), y_{1}(\cdot)\right)-\left(u_{2}(\cdot), y_{2}(\cdot)\right)\right\|_{\mathscr{H}} .
\end{aligned}
$$

Thus, the introduced operator $\tilde{P}$ is a nonexpansive operator in the Hilbert space $\mathscr{H}$. By Lemma 2.4, $\mathfrak{U}_{l}^{f} \times \mathbb{L}_{n}^{2}\left(0, t_{f}\right)$ is a nonempty closed and convex subset of $\mathscr{H}$. Finally, from Proposition 2.3, it follows that $F(\widetilde{P})$ is a nonempty closed and convex set. The proof is completed.

Note that Theorem 3.1 establishes the convexity property of the set of fixed points for the extended system operator $\tilde{P}$ on $\mathcal{U}_{l}^{f} \times \mathbb{L}_{n}^{2}\left(0, t_{f}\right)$ (see (2.6) and (2.5)). As a consequence of this 
result, we also can formulate the corresponding theorem for the operator $P$ on $\mathcal{U}_{l}^{f} \times \mathbb{W}_{n}^{1,1}\left(0, t_{f}\right)$ (see (2.4) and (2.5)).

Theorem 3.2. Under the assumption of Theorem 3.1, the set $F(P)$ is convex. Moreover, the set of trajectories $\tau\left(x_{0}\right)=\left\{x^{u}(\cdot) \mid u(\cdot) \in \mathcal{U}_{l}^{f}\right\}$ for the initial value problem (1.1) on $\left[0, t_{f}\right]$ is also convex.

Proof. Since $f$ is a Lipschitz continuous function, the initial value problem (1.1) has a solution and the set $F(P)$ of fixed points of the operator $P$ is nonempty. By Proposition 2.1, we have $\mathbb{W}_{n}^{1,1}\left(0, t_{f}\right) \subset \mathbb{L}_{n}^{2}\left(0, t_{f}\right)$. Hence,

$$
F(P)=\mathbb{W}_{n}^{1,1}\left(0, t_{f}\right) \bigcap F(\tilde{P})
$$

Since $\mathbb{W}_{n}^{1,1}\left(0, t_{f}\right)$ is a convex subset of $\mathbb{L}_{n}^{2}\left(0, t_{f}\right)$, the set $F(P)$ is also convex.

In fact, $F(P)$ is a subset of the product-space $\mathcal{U}_{l}^{f} \times \mathbb{W}_{n}^{1,1}\left(0, t_{f}\right)$ and the structure of the operator $P$ defines the structure of the set $F(P)=\mathfrak{U}_{l}^{f} \times \mathcal{\tau}\left(x_{0}\right)$. Since $F(P)$ and $\mathfrak{U}_{l}^{f}$ are convex, we obtain the convexity of the set $\tau\left(x_{0}\right)$.

We now deal with the reachable set $\mathcal{K}\left(t, x_{0}\right)$ for the closed-loop system (1.1). Our next result is an immediate consequence of the convexity criterion just presented in Theorem 3.2.

Theorem 3.3. Under the assumption of Theorem 3.1, the reachable set $\mathcal{K}\left(t, x_{0}\right)$ for the initial value problem (1.1) is convex for every $t \in\left[0, t_{f}\right]$.

Proof. Theorem 3.2 states the convexity of the set $\tau\left(x_{0}\right)$. It means that for

$$
x^{u_{1}}(\cdot), x^{u_{2}}(\cdot) \in \tau\left(x_{0}\right)
$$

with $u_{1}(\cdot), u_{2}(\cdot) \in \mathcal{U}_{l}^{f}$ and for fixed $\lambda \in(0,1)$, there exists an admissible control $u_{3}(\cdot) \in \mathcal{U}_{l}^{f}$ such that $x^{u_{3}}(\cdot)=\lambda x^{u_{1}}(\cdot)+(1-\lambda) x^{u_{2}}(\cdot) \in \tau\left(x_{0}\right)$. On the other hand, at a time-instant $t \in\left[0, t_{f}\right]$, we have $x^{u_{1}}(t), x^{u_{2}}(t) \in \mathcal{K}\left(t, x_{0}\right)$. Hence,

$$
x^{u_{3}}(t)=\lambda x^{u_{1}}(t)+(1-\lambda) x^{u_{2}}(t) \in \mathcal{K}\left(t, x_{0}\right) .
$$

This shows that the reachable set $\mathcal{K}\left(t, x_{0}\right)$ is convex for every $t \in\left[0, t_{f}\right]$.

Remark 3.4. Note that under the conditions of Theorem 3.1, the reachable set $K\left(t, x_{0}\right)$ from Theorem 3.3 is closed for every $t \in\left[0, t_{f}\right]$. This fact also follows from Proposition 2.3 and Theorem 3.1. Moreover, the set of trajectories $\tau\left(x_{0}\right)$ of (1.1) is a closed subset of the space $\mathbb{C}\left(0, t_{f}\right)[14]$.

We now present two illustrative examples of control systems (1.1) satisfying the Lipschitz conditions from the main Theorem 3.1. 
Example 3.5. Let us consider an $f$ with every component $f_{j}$ being a convex function $f_{i}: \in$ $U \times \mathbb{R}^{n} \rightarrow \mathbb{R}$. In case $m_{j} \leq f_{j}(x, u) \leq M_{j}, j=1, \ldots, n$ holds for all $(u, x)$ in the ball $\mathbb{B}(0,2 \Delta)$ of radius $2 \Delta$ around 0 , every $f_{j}$ is Lipschitzian on $B(0, \Delta)$ with

$$
\left|f_{j}\left(x_{1}, u_{1}\right)-f_{j}\left(x_{2}, u_{2}\right)\right| \leq \frac{M_{j}-m_{j}}{\Delta}
$$

for all $\left(u_{1}, x_{1}\right),\left(u_{2}, x_{2}\right) \in \mathbb{B}(0, \Delta)$ (cf. [15]) implying that

$$
\begin{gathered}
v:=\max _{j=1, \ldots, n} \frac{\left(M_{j}-m_{j}\right)}{\Delta} \\
\left|f\left(x_{1}, u_{1}\right)-f\left(x_{2}, u_{2}\right)\right| \leq v\left(\left\|u_{1}-u_{2}\right\|+\left\|x_{1}-x_{2}\right\|\right) .
\end{gathered}
$$

Therefore, the condition $t_{f} \sqrt{l^{2}+\left(l_{1}+l_{2} l\right)^{2}} \leq 1$ from Theorem 3.1 can be written as follows:

$$
t_{f} \sqrt{l^{2}+v^{2}(1+l)^{2}} \leq 1
$$

where $B(0, \Delta)$ is taken for $\Omega$. Note that $M_{j}$ and $m_{j}$ may depend on $\Delta$ too.

Example 3.6. Consider the following two-dimensional control system:

$$
\begin{gathered}
\dot{x}_{1}(t)=x_{2}(t), \\
\dot{x}_{2}(t)=-\sin x_{1}(t)+u(x(t)), \\
x_{1}(0)=x_{2}(0)=0,
\end{gathered}
$$

where $x:=\left(x_{1}, x_{2}\right)^{T}$ and $t \in[0,0.5]$. It is easy to see that $l_{1}=l_{2}=1$. The condition $t_{f} \sqrt{l^{2}+\left(l_{1}+l_{2} l\right)^{2}} \leq 1$ from Theorem 3.1 implies $2 l^{2}+2 l-3 \leq 0$ and

$$
0<l \leq \frac{\sqrt{7}-1}{2}
$$

We see that under this condition the reachable set $\mathcal{K}\left(t, 0_{\mathbb{R}^{2}}\right)$ of the presented system is convex for every $t \in[0,0.5]$.

\section{Overapproximations of Reachable Sets}

In this section we will discuss a special class of closed-loop and open-loop systems (1.1) and (1.5), namely, systems which satisfy the following condition:

$$
f(x, u), g(x, u) \in K, \quad \forall(x, u) \in \mathbb{R}^{n} \times U,
$$


where $K$ is a closed convex subset of $\mathbb{R}^{n}$ containing 0 . The right-hand sides $f$ and $g$ of (1.1) and (1.5) are assumed to be continuous in both components. Let us first formulate the following auxiliary abstract result.

Lemma 4.1. Let $\mathcal{X}$ be a separable Banach space and $\{\mathcal{S}, \Sigma, \mu\}$ be a measurable space with a probability measure $\mu$. Let $C \subset \mathcal{X}$ be closed and convex. If the mapping $q: S \rightarrow C$ is a $\mu$-measurable function, then

$$
\int q(\tau) \mu(d \tau) \in C
$$

Proof. Assume $a:=\int q(\tau) \mu(d \tau) \notin C$ and let

$$
B(a, R):=\left\{\xi \in \mathcal{X} \mid\|\xi-a\|_{\mathcal{X}}<R\right\}
$$

be the ball around $a$ with radius $R$. Evidently, there is a radius $R$ such that we have $C \cap$ $B(a, R)=\emptyset$. Using a Separating Theorem from convex analysis (cf. [13, 16]), we obtain a nontrivial $\mathcal{L} \in X^{*}$ with $\mathcal{L} \xi \leq \mathcal{L} \psi$ for all $\xi \in B(a, R), \psi \in C$. By $X^{*}$, we have denoted the (topological) dual space to $\mathcal{X}$. Thereby, we have the inequality

$$
\sup _{\|\xi\|_{\chi} \leq 1}(\mathcal{L}(a+R \xi))=\mathcal{L} a+R|\mathcal{L}| \leq \mathcal{L}(q(\tau)), \quad \tau \in \mathcal{S},
$$

and-by integration with respect to $\mu$-we have also the corresponding inequality

$$
\mathcal{L} a+R|\mathcal{L}| \leq \int \mathcal{L}(q(\tau)) \mu(d \tau)
$$

Because of

$$
\int \mathcal{L}(q(\tau)) \mu(d \tau)=\mathcal{\perp} \int q(\tau) \mu(d \tau)=\llcorner a
$$

(4.5) leads to $\mathcal{L} a+R|\mathcal{L}| \leq \mathcal{L}$ contradicting the fact that $\mathcal{L}$ is nontrivial. Therefore,

$$
a=\int q(\tau) \mu(d \tau)
$$

belongs to $C$.

Returning to control systems of type (1.1) or (1.5) satisfying condition (4.1), we introduce the following Lebesgue probability measure $\mu=\tau / t$ on the interval $[0, t]$. Then, we apply Lemma 4.1 to our control systems and compute the state of system (1.1) (or the state of system (1.5)) at time $t \in\left[0, t_{f}\right]$ as

$$
x(t)=x_{0}+\int_{0}^{t} f(x(\tau), u(x(\tau))) d s=x_{0}+t \int_{0}^{t} f(x(\tau), u(x(\tau))) \mu(d \tau) \in x_{0}+t K .
$$


For the open-loop system (1.5), we have the analogous result

$$
x(t)=x_{0}+\int_{0}^{t} g(x(\tau), u(\tau)) d \tau \in x_{0}+t K
$$

This means that the reachable sets of systems (1.1) and (1.5) with initial value $x_{0}$ belong to the closed convex set $\Omega:=x_{0}+t_{f} K$. Since $x_{0}$ belongs to $\Omega$, we have the set $\Omega$ as a positively invariant set for the corresponding control system. In particular, this set $\Omega$ contains the reachable set of the considered dynamical system.

We now describe an abstract approach for estimating convex reachable sets. Our main idea is as follows: under the assumption of convexity for the reachable set of a given closedloop control system, we formulate an auxiliary feedback optimal control problem with a linear cost functional. A solution of this problem makes it possible to construct a tangent hyperplane (supporting hyperplane) to the reachable set under consideration. Considering a sufficiently "rich" set of these hyperplanes and their intersections, one can approximate the reachable set with arbitrary accuracy.

Let $\mathcal{K}\left(\widehat{t}, x_{0}\right), \widehat{t} \in\left[0, t_{f}\right]$ be a bounded closed and convex reachable set for (1.1). Following the idea sketched above, let us consider the auxiliary optimal feedback control problem

$$
\operatorname{minimize}\langle c, x(\hat{t})\rangle
$$

subject to (1.1),

where $c$ is a fixed vector from $\mathbb{R}^{n}, u(\cdot) \in \mathcal{U}_{l}^{f}$, and $\langle\cdot, \cdot\rangle$ denotes the scalar product in $\mathbb{R}^{n}$. Note that (4.10) is formulated as a minimizing problem with respect to a terminal linear cost functional. Linearity of this cost functional and the above properties of the reachable set $\mathcal{K}\left(\widehat{t}, x_{0}\right)$ to the time $\widehat{t} \in\left[0, t_{f}\right]$ imply the existence of an optimal solution $\left(u^{*}(\cdot), x^{*}(\cdot)\right)$ for (4.10) (see [16]), where $u^{*}(\cdot) \in \mathcal{U}_{l}^{f}$. Note that (4.10) can be reformulated as the following convex linear problem in $\mathbb{R}^{n}$ :

$$
\begin{gathered}
\operatorname{minimize}\langle c, z\rangle \\
\text { subject to } z \in \mathcal{K}\left(\widehat{t}, x_{0}\right) .
\end{gathered}
$$

Therefore, $x^{*}(\widehat{t}) \in \partial \mathcal{K}\left(\widehat{t}, x_{0}\right)$, where $\partial \mathcal{K}\left(\widehat{t}, x_{0}\right)$ is the boundary (the set of all extremal points) of the convex set $\mathcal{K}\left(\hat{t}, x_{0}\right)$ (see, e.g., $\left.[15,16]\right)$.

We now recall the Rademacher Theorem (see, e.g., [17]), which states that a function which is Lipschitz on an open subset of $\mathbb{R}^{n}$ is differentiable almost everywhere (in the sense of a Lebesgue measure) on that subset. Since $\Omega \subset \mathbb{R}^{n}$ is an open set, the function $u^{*}(\cdot)$ is differentiable almost everywhere on $\Omega$. The set of points at which the optimal control $u^{*}(\cdot)$ fails to be differentiable is denoted $\Omega^{0}$. Evidently meas $\left(\Omega^{0}\right)=0$. Let $\Omega^{*}:=\Omega \backslash \Omega^{0}$. We now formulate our next hypotheses.

(H2) The right-hand side $f(\cdot, \cdot)$ of (1.1) is a differentiable function (in both components) such that the partial derivatives $f_{x}(\cdot, \cdot), f_{u}(\cdot, \cdot)$ are integrable functions on $\Omega \times U$. 
(H3) It hold that $x^{*}(t) \in \Omega^{*}$ for all $t \in[0, \widehat{t}]$ and the derivative $\left(u^{*}\right)^{\prime}$ of $u^{*}(\cdot)$ is locally integrable on $\Omega^{*}$.

Clearly, the optimal control problem (4.10) is equivalent to the following minimization problem:

$$
\operatorname{minimize}\left(c,\left[x_{0}+\int_{0}^{\hat{t}} f(x(t), u(x(t))) d t\right]\right)
$$

for $u(\cdot) \in \mathcal{U}_{l}^{f}$. Since the right-hand side of the differential equation from (1.1) is supposed to be differentiable in both components, the cost functional in (4.12) is Fréchet differentiable (see, e.g., [18]). Assume (H2)-(H3) and formulate the necessary optimality condition for $\left(u^{*}(\cdot), x^{*}(\cdot)\right)$ to be an optimal solution of $(4.12)$ :

$$
\begin{array}{r}
\left.D\left(c, x^{*}(\hat{t})\right)\right|_{y(\cdot)}=\left(c, \int_{0}^{\hat{t}}\left[f_{x}\left(x^{*}(t), u^{*}\left(x^{*}(t)\right)\right)+f_{u}\left(x^{*}(t), u^{*}\left(x^{*}(t)\right)\right)\left(u^{*}\right)^{\prime}\left(x^{*}(t)\right)\right] y(t) d t\right)=0 \\
\forall y(\cdot) \in \mathbb{W}_{n}^{1,1}(0, \hat{t}),
\end{array}
$$

where $\left.D\left(c, x^{*}(\widehat{t})\right)\right|_{y(\cdot)}$ is the Fréchet derivative of the cost functional from (4.12) at $x^{*}(\cdot)$. Note that under the above assumptions ( $\mathrm{H} 2)-(\mathrm{H} 3)$, the integrand in (4.13) is a locally integrable function. Moreover, (4.13) holds for all functions $y(\cdot)$ from the space $\mathbb{W}_{n}^{1,1}(0, \widehat{t})$. Therefore, the expression in (4.13) is also equal to zero for all functions $y(\cdot)$ from $\mathbb{C}_{0}^{\infty}(0, \widehat{t})$, where

$$
\mathbb{C}_{0}^{\infty}(0, \hat{t}):=\left\{\xi(\cdot) \in \mathbb{C}^{\infty}(0, \hat{t}) \mid \operatorname{supp}\{\xi(\cdot)\} \text { is a compact subset of }(0, \widehat{t})\right\}
$$

and $\operatorname{supp}\{\xi(\cdot)\}:=\{t \in(0, \widehat{t}) \mid \xi(t) \neq 0\}$. By the Generalized Variational Lemma (see e.g., [6, Lemma 7.1.2]), we deduce from (4.13) that

$$
\left[f_{x}\left(x^{*}(t), u^{*}\left(x^{*}(t)\right)\right)+f_{u}\left(x^{*}(t), u^{*}\left(x^{*}(t)\right)\right)\left(u^{*}\right)^{\prime}\left(x^{*}(t)\right)\right] c=0 \quad \text { a.e. on }(0, \hat{t})
$$

The nonlinear equation (4.15) with a given vector $c \in \mathbb{R}^{n}$ provides a basis for solving optimal control problem (4.10).

Consider now an interior point $\zeta$ of the convex hull $\operatorname{conv}\{\bar{\Omega}\}$ and a family $\left\{z_{s}\right\}$ of elements $z_{s} \in \partial \operatorname{conv}\{\bar{\Omega}\}, s=1, \ldots, S$, for a sufficiently large number $S \in \mathbb{N}$ such that $\left\{z_{s}\right\}$ approximate the boundary $\partial \operatorname{conv}\{\bar{\Omega}\}$ of $\operatorname{conv} \bar{\Omega}$. By $\bar{\Omega}$, we denote here the closure of $\Omega$. If we solve the family of problems (4.10) with $c_{s}:=z_{s}-\zeta, s=1, \ldots, S$, we obtain the corresponding optimal state vectors $x_{s}^{*}(\widehat{t})$. As established above, $x_{s}^{*}(\widehat{t}) \in \partial \mathcal{K}\left(\widehat{t}, x_{0}\right)$. Therefore, we can write the equation of the approximating tangent hyperplane $T_{s}$ to the reachable set at $x_{s}^{*}(\widehat{t})$ in the form

$$
T_{s}:=\left\{x \in \mathbb{R}^{n} \mid c_{s}, x-x_{s}^{*}(\hat{t})=0\right\} .
$$


If we examine all hyperplanes $T_{s}, s=1, \ldots, S$ and their intersections, we can constract a convex polyhedron which contains the reachable set $\mathcal{K}\left(\widehat{t}, x_{0}\right)$. In principle, the proposed idea guarantees an overapproximation for a convex reachable set of a control system (1.1). However, it is necessary to stress that complexity of this approximation grows rapidly if we increase the number $S \in \mathbb{N}$. Finally, note that the same idea can also be used for the overapproximations of reachable sets for open-loop control systems. We refer to [10] for details.

\section{An Application to Optimal Control Problems with Constraints}

Let us now apply the main convexity result of Theorem 3.2 to the following constrained optimal feedback control problem:

$$
\begin{gathered}
\text { minimize } J(x(\cdot)) \\
\text { subject to }(1.1), \quad x(\cdot) \in A \text {, }
\end{gathered}
$$

where $J$ is a bounded, convex, and lower semicontinuous objective functional (see [16]) and $A$ a nonempty, bounded, closed, and convex subset of $\mathbb{L}_{n}^{2}\left(0, t_{f}\right)$. The given control system (1.1) is supposed to satisfy the conditions of Theorem 3.1. We consider the optimal control problem (5.1) on the Hilbert space $\mathbb{L}_{n}^{2}\left(0, t_{f}\right)$ with feedback controls from $\mathcal{U}_{l}^{f}$. Note that the class of feedback optimal control problems of type (5.1) is quite general [3]. For example, the objective functional $J$ could be given by

$$
J(x(\cdot))=\int_{0}^{t_{f}} x^{2}(t) d t
$$

and the abstract restriction $x(\cdot) \in A$ could arise from a system of the following inequalities $h_{s}(x(t)) \leq 0$ for all $t \in\left[0, t_{f}\right]$ with convex functions $h_{s}: \mathbb{R}^{n} \rightarrow \mathbb{R}$, where $s=1, \ldots, S$. It is clear that an optimal control problem does not always have a solution. The question of existence of an optimal feedback solution is generally a delicate one (cf. [3]).

Let $\tau\left(x_{0}\right) \cap A$ be nonempty. Evidently, problem (5.1) can be rewritten as an optimization problem over the set $\mathcal{C}:=\tau\left(x_{0}\right) \cap A$ of admissible trajectories as follows:

$$
\begin{aligned}
& \text { minimize } J(x(\cdot)) \\
& \text { subject to } x(\cdot) \in \mathcal{C} \text {. }
\end{aligned}
$$

Here, the state (1.1) is included into the constraints $x(\cdot) \in \mathcal{C}$. We claim that (5.3) is a standard convex optimization problem on a bounded closed convex subset of a Hilbert space (see, e.g., [16]). To see this, we note that the set of solutions:

$$
\tau\left(x_{0}\right)=\left\{x^{u}(\cdot) \mid u(\cdot) \in \mathcal{U}_{l}^{f}\right\}
$$

is a closed subset of the space $\mathbb{C}\left(0, t_{f}\right)$ (see [14]). Therefore, this set is also closed in the sense of the norm of $\mathbb{L}_{n}^{2}\left(0, t_{f}\right)$. Moreover, $\mathcal{\tau}\left(x_{0}\right)$ is convex by Theorem 3.2. The intersection $\mathcal{C}$ of 
the two closed convex sets $A$ and $\tau\left(x_{0}\right)$ is again closed and convex set in the Hilbert space $\mathbb{L}_{n}^{2}\left(0, t_{f}\right)$. Since $A$ is bounded, the set $\mathcal{C}$ is also bounded. Using the well-known existence results from convex optimization theory (cf. [16]), one can establish the following existence result for optimal control problem (5.1).

Theorem 5.1. Under the conditions of Theorem 3.2, the optimal control problem (5.3) with a bounded convex and lower semicontinuous objective functional J and bounded closed convex set $A$ has at least one optimal solution

$$
\left(u^{\mathrm{opt}}(\cdot), x^{\mathrm{opt}}(\cdot)\right) \in \mathcal{U}_{l}^{f} \times \mathbb{L}_{n}^{2}\left(0, t_{f}\right)
$$

provided that $\mathcal{C}=\tau\left(x_{0}\right) \cap A$ is nonempty.

Since $\mathbb{W}_{n}^{1,1}\left(0, t_{f}\right) \subset \mathbb{L}_{n}^{2}\left(0, t_{f}\right)$ and $\mathbb{W}_{n}^{1,1}\left(0, t_{f}\right)$ is convex, the following intersection $\mathbb{W}_{n}^{1,1}\left(0, t_{f}\right) \cap \mathcal{C}$ is also a bounded closed and convex subset of $\mathbb{L}_{n}^{2}\left(0, t_{f}\right)$. Therefore, we also obtain the corresponding existence result for problem (5.1) considered on the space $\mathbb{W}_{n}^{1,1}\left(0, t_{f}\right)$. Let $\left\{x^{k}(\cdot)\right\} \subset \mathbb{W}_{n}^{1,1}\left(0, t_{f}\right)$ be a minimizing sequence for (5.3) defined on the space $\mathbb{W}_{n}^{1,1}\left(0, t_{f}\right)$, that is,

$$
\lim _{k \rightarrow \infty} J\left(x^{k}(\cdot)\right)=\min _{\mathbb{W}_{n}^{1,1}\left(0, t_{f}\right) \cap C} J(x(\cdot))
$$

It is well known that a minimizing sequence does not always converge to an optimal solution. The question of creating a convergent minimizing sequence is a central question in the numerical analysis of optimization algorithms (see, e.g., [11, 19]). By Proposition 2.1, each bounded sequence in $\mathbb{W}_{n}^{1,1}\left(0, t_{f}\right)$ has a convergent subsequence in $\mathbb{L}_{n}^{2}\left(0, t_{f}\right)$. Since $\left\{x^{k}(\cdot)\right\}$ is bounded, we have

$$
\lim _{l \rightarrow \infty}\left\|x^{k_{l}}(\cdot)-\underset{\mathbb{W}_{n}^{1,1}\left(0, t_{f}\right) \cap \mathcal{C}}{\operatorname{Argmin}} J(x(\cdot))\right\|_{\mathbb{L}_{n}^{2}\left(0, t_{f}\right)}=0
$$

for a subsequence $\left\{x^{k_{l}}(\cdot)\right\}$ of $\left\{x^{k}(\cdot)\right\}$. Thus, by Theorem 5.1, we deduce the existence of an $\mathbb{L}_{n}^{2}$-convergent minimizing sequence $\left\{x^{k_{l}}(\cdot)\right\}$ for the optimal control problem (5.3).

\section{Conclusion}

In this paper, we proposed a new convexity criterion for reachable sets for a class of closed-loop control systems. This sufficient condition is based on a general convexity result for solution sets of the corresponding nonlinear dynamical systems. Convexity of the set of trajectories makes it also possible to study some constrained feedback optimal control problems. For some families of closed-loop and open-loop control systems, we construct an overestimation of the examined reachable set, that is, we provide sets that contain the reachable sets of the dynamical system under consideration. 


\section{References}

[1] E. Zeidler, Nonlinear Functional Analysis and Its Applications. I: Fixed-Point Theorems, Springer, New York, NY, USA, 1990.

[2] W. Takahashi, Nonlinear Functional Analysis, Yokohama Publishers, Yokohama, Japan, 2000.

[3] L. D. Berkovitz, Optimal Control Theory, vol. 1, Springer, New York, NY, USA, 1974.

[4] H. O. Fattorini, Infinite-Dimensional Optimization and Control Theory, vol. 62 of Encyclopedia of Mathematics and Its Applications, Cambridge University Press, Cambridge, UK, 1999.

[5] R. A. Adams, Sobolev Spaces, vol. 65 of Pure and Applied Mathematics, Academic Press, New York, NY, USA, 1975.

[6] K. Atkinson and W. Han, Theoretical Numerical Analysis, vol. 39 of Texts in Applied Mathematics, Springer, New York, NY, USA, 2nd edition, 2005.

[7] E. D. Sontag, Mathematical Control Theory, vol. 6 of Texts in Applied Mathematics, Springer, New York, NY, USA, 2nd edition, 1998.

[8] A. B. Kurzhanski and P. Varaiya, "Ellipsoidal techniques for reachability analysis," in Hybrid Systems: Computation and Control, vol. 1790 of Lecture Notes in Computer Science, pp. 202-214, Springer, New York, NY, USA, 2000.

[9] B. T. Polyak, S. A. Nazin, C. Durieu, and E. Walter, “Ellipsoidal parameter or state estimation under model uncertainty," Automatica, vol. 40, no. 7, pp. 1171-1179, 2004.

[10] B. T. Polyak, "Convexity of the reachable set of nonlinear systems under $L_{2}$ bounded controls," Dynamics of Continuous, Discrete E Impulsive Systems. Series A, vol. 11, no. 2-3, pp. 255-267, 2004.

[11] V. Azhmyakov, "A numerically stable method for convex optimal control problems," Journal of Nonlinear and Convex Analysis, vol. 5, no. 1, pp. 1-18, 2004.

[12] E. H. Zarantonello, "Projections on convex sets in Hilbert space and spectral theory. I. Projections on convex sets," in Contributions to Nonlinear Functional Analysis, pp. 237-341, Academic Press, New York, NY, USA, 1971.

[13] C. D. Aliprantis and K. C. Border, Infinite-Dimensional Analysis, Springer, Berlin, Germany, 2nd edition, 1999.

[14] A. F. Filippov, Differential Equations with Discontinuous Righthand Sides, vol. 18 of Mathematics and Its Applications (Soviet Series), Kluwer Academic Publishers, Dordrecht, The Netherlands, 1988.

[15] J.-B. Hiriart-Urruty and C. Lemaréchal, Fundamentals of Convex Analysis, Grundlehren Text Editions, Springer, Berlin, Germany, 2001.

[16] I. Ekeland and R. Temam, Convex Analysis and Variational Problems, Studies in Mathematics and Its Applications, North-Holland, Amsterdam, The Netherlands, 1976.

[17] F. H. Clarke, Optimization and Nonsmooth Analysis, vol. 5 of Classics in Applied Mathematics, SIAM, Philadelphia, Pa, USA, 2nd edition, 1990.

[18] A. D. Ioffe and V. M. Tihomirov, Theory of Extremal Problems, vol. 6 of Studies in Mathematics and Its Applications, North-Holland, Amsterdam, The Netherlands, 1979.

[19] V. Azhmyakov, V. G. Boltyanski, and A. Poznyak, "Optimal control of impulsive hybrid systems," Nonlinear Analysis: Hybrid Systems, vol. 2, no. 4, pp. 1089-1097, 2008. 


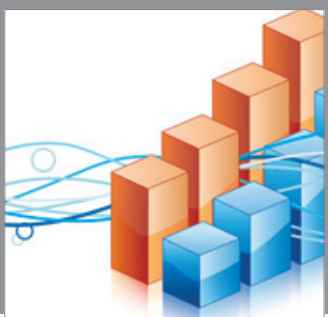

Advances in

Operations Research

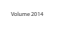

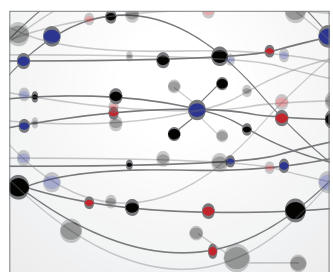

\section{The Scientific} World Journal
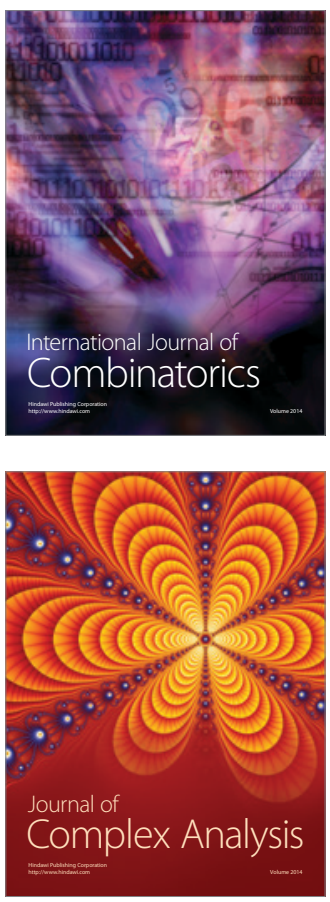

International Journal of

Mathematics and

Mathematical

Sciences
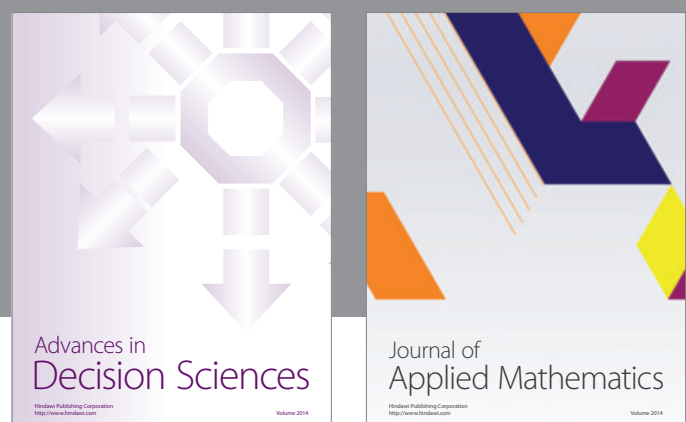

Journal of

Applied Mathematics
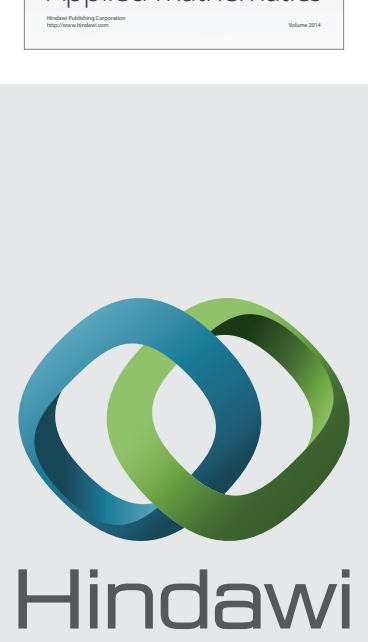

Submit your manuscripts at http://www.hindawi.com
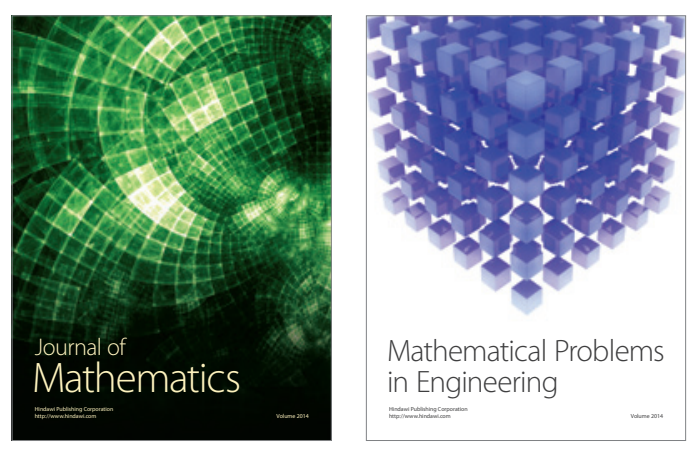

Mathematical Problems in Engineering
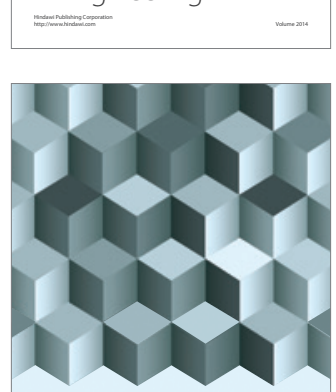

Journal of

Function Spaces
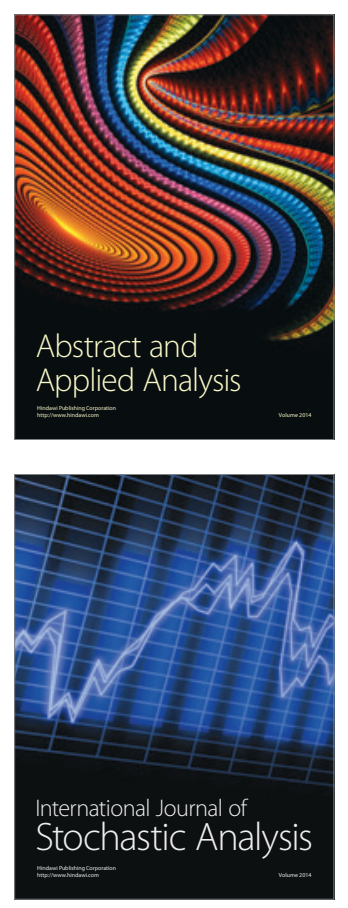

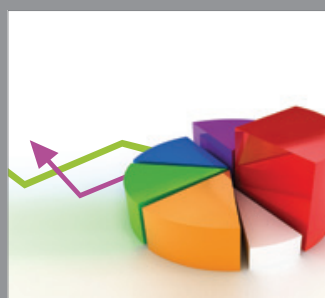

ournal of

Probability and Statistics

Promensencen
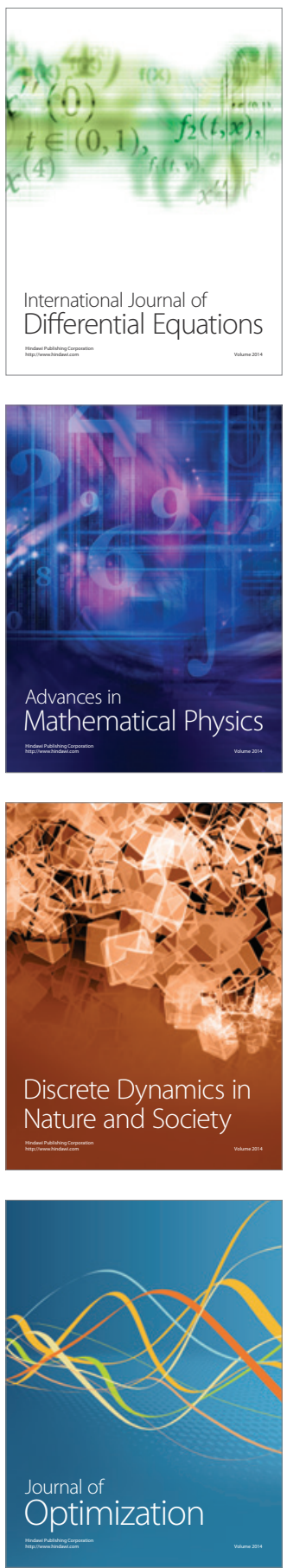